\title{
Receta para una convivencia fiscal sana y saludable
}

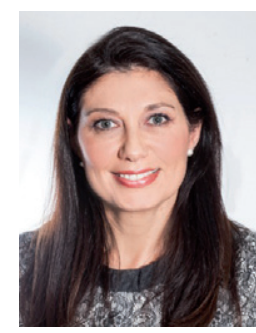

Virginia Rey Paredes Especialista en tributación. Profesora en el Departamento de Economía. Universidad CEU San Pablo. Madrid. Profesora en el Máster de Fiscalidad Internacional. Universidad Internacional de la Rioja.

Profesora en el Máster en Mercados Financieros y Gestión de Activos. Instituto de Estudios Bursátiles. Madrid.

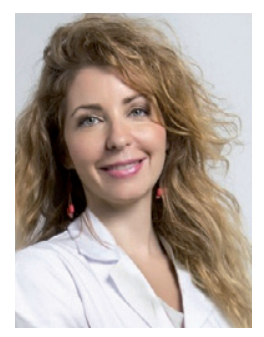

Elena González-Guerra Médico adjunto del Servicio de Dermatología. Hospital Universitario Clínico San Carlos. Madrid. Profesora asociada de la Universidad Complutense de Madrid.

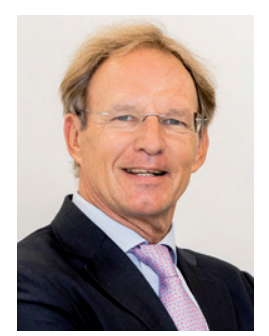

Ricardo Palomo Zurdo Decano de la Facultad de Ciencias Económicas y Empresariales. Director de CEU Digital. Catedrático de Economía Financiera.

Universidad CEU San Pablo. Madrid.
Convivir con la fiscalidad es una expresión que puede incluir cierto sentido eufemístico, pues es sabido que toda tributación implica una merma en los ingresos netos percibidos por los contribuyentes; si bien, la carga impositiva que soportan empresas, familias y profesionales puede ser justificable con las prestaciones de toda índole que se perciben de las administraciones nacional, autonómica o local y que financian el «casi todo incluido» en términos similares a la cuota de una comunidad de vecinos: pagamos piscina si la tiene, aunque no la utilicemos.

Sin entrar en la justificación, el debate o la controversia de la finalidad o la equidad de los conceptos impositivos, lo cierto es que el pago de impuestos por el ejercicio de una actividad empresarial o profesional se remonta a las primeras civilizaciones mesopotámicas; desde el primer momento en que debe mantenerse una estructura administrativa en todas sus facetas, como las obras públicas o las prestaciones asistenciales, educativas o de defensa.

El personal sanitario, reconocido por su aportación indudable de valor a la sociedad e integrado por una amplia y heterogénea base de profesionales, está sometido, como cualquier otra profesión, a un régimen fiscal que, en ocasiones, complica su entendimiento.

Es habitual que, además, en un amplio sector de los profesionales sanitarios, concurran circunstancias que hacen más compleja la situación y que vamos a tratar de explicar seguidamente del modo más sencillo y claro posible.

La posible doble dimensión de muchos profesionales de la sanidad se debe a una idiosincrasia particular del sector, debida a una combinación de la figura del trabajador por cuenta ajena para una entidad sanitaria (hospital, centro de salud, clínica, etc.) o como docente, junto con la figura del trabajador por cuenta 
propia por el ejercicio de actividades profesionales bajo el régimen de trabajador autónomo.

Por lo tanto, el primer punto que se ha de abordar es tratar de distinguir entre si el personal sanitario desarrolla su actividad por cuenta ajena (normalmente, bajo contrato laboral), o bien si desarrolla su actividad por cuenta propia (contrato mercantil, autónomo...).

\section{ACTIVIDAD DE PROFESIONALES DE LA SALUD DESARROLLADA POR CUENTA AJENA}

En el caso de desarrollar su actividad por cuenta ajena o con contrato laboral, los rendimientos obtenidos tienen la calificación fiscal o el tratamiento de rendimientos del trabajo. Este es el supuesto habitual de los trabadores del sistema público de salud; o bien, cuando se perciben salarios por impartir docencia, esto es, cuando desarrolla su labor como profesor universitario, entre otras actividades, o bien, cuando desarrolla su actividad para un hospital, clínica, etc., pero con contrato laboral. Estos rendimientos obtenidos a lo largo del año han de ser declarados en su Impuesto sobre la Renta de las Personas Físicas (IRPF) como rendimientos del trabajo por el importe íntegro obtenido. En este caso, el importe es sencillo de conocer, puesto que el empleador (empresa que le ha contratado) le proporcionará un certificado de retenciones, en el cual aparecerán reflejados el importe íntegro obtenido, el importe de las retenciones que, en concepto de IRPF, le han ido descontando de la nómina, así como el importe de las cotizaciones a la Seguridad Social, que, igualmente, le habrán sido descontadas de cada mensualidad.

Llegados a este punto, debemos detenernos en una cuestión que muchas veces genera dudas sobre cuándo han de ser declaradas; se trata de las «guardias» médicas, puesto que estas son abonadas e incluidas en la nómina en el mes siguiente a su realización. La problemática se plantea con las guardias médicas realizadas durante el mes de diciembre, pues son incluidas en la nómina del mes de enero del año siguiente. En este caso, se incluyen como rendimientos del año siguiente, puesto que la regla general de cobro de las guardias es el mes siguiente a su realización, y no se computan como si se tratara del cobro de un atraso (de ser un atraso, se imputarían las cantidades al mes en que surge su derecho por haberse realizado y no al mes del cobro efectivo). La normativa del IRPF indica que los rendimientos se han de declarar en el ejercicio en que sean exigibles; y las guardias médicas realizadas en el mes de diciembre no son exigibles en diciembre, sino en el mes de enero del año siguiente, correspondiendo, por lo tanto, al siguiente ejercicio fiscal y debiendo ser declaradas en ese año siguiente a su realización.

En el IRPF, el ejercicio fiscal coincide con el año natural, esto es, desde el 1 de enero al 31 de diciembre, devengándose el impuesto el 31 de diciembre, último día del ejercicio fiscal, salvo fallecimiento, por lo que es entendible que no haya tiempo material para la contabilización y comunicación a los departamentos de nóminas antes del cierre del ejercicio.

Una vez determinados los ingresos que se han de declarar bajo la categoría de rendimientos del trabajo, se ha de establecer qué gastos son fiscalmente deducibles. En este sentido, los gastos deducibles vienen recogidos en el artículo 19 de la Ley del IRPF, entre los cuales, cabe destacar los siguientes, por ser los más habituales para la profesión sanitaria:

- Las cotizaciones a la Seguridad Social o a mutualidades generales obligatorias de funcionarios.

- Las cotizaciones a los colegios de huérfanos o entidades similares.

- Las cuotas satisfechas a sindicatos.

- Las cuotas satisfechas a los colegios profesionales, cuando la colegiación tenga carácter obligatorio para el desempeño del trabajo, en la parte que corresponda a los fines esenciales de estas instituciones, con el límite de 500 euros anuales (artículo 10 del Reglamento del IRPF). 
- Los gastos de defensa jurídica derivados directamente de litigios suscitados en la relación del contribuyente con la persona de la que percibe los rendimientos, con el límite de 300 euros anuales.

- Otros gastos: la norma crea una reducción general de 2000 euros, que se incrementa en el caso de discapacidad del trabajador.

Una vez que se conocen tanto los ingresos percibidos como los gastos deducibles generados en el ejercicio, se determina el rendimiento neto del trabajo y el importe resultante se incluye en la base imponible general del IRPF (junto con el resto de otros rendimientos), tributando a una escala progresiva, que abarca desde un mínimo del $19 \%$ hasta un máximo del $47 \%$, en función del nivel global de rentas de cada contribuyente.

\section{ACTIVIDAD DE PROFESIONALES DE LA SALUD DESARROLLADA POR CUENTA PROPIA O CON CONTRATO MERCANTIL}

Por lo que respecta al supuesto de los profesionales de la salud que desarrollan su actividad por cuenta propia (coloquialmente, conocido como «ser autónomo»), o con contrato mercantil, las obligaciones fiscales y los rendimientos se calculan del modo que se detalla a continuación.

En el supuesto de actuar por cuenta propia (como autónomo), lo primero que se debe tener en cuenta es que la Agencia Tributaria considera que sus rendimientos tienen la consideración de actividad económica (como cualquier otro empresario o profesional persona física). La actual legislación indica que se consideran rendimientos de actividades económicas aquellos que «procediendo del trabajo personal y del capital conjuntamente, o de uno de estos factores, supongan, por parte del contribuyente, la ordenación por cuenta propia de medios de producción y de recursos humanos o de uno de ambos, con la finalidad de intervenir en la producción o distribución de bienes o servicios».
Como profesional por cuenta propia, la primera obligación es darse de alta en la declaración censal (modelo 036) ante la Agencia Tributaria, indicando el momento en que se comenzarán a prestar los servicios. Esta comunicación se realiza en los plazos establecidos en la normativa y, como muy tarde, el día anterior al del inicio de la actividad.

En cuanto a las obligaciones contables, al ser profesional (estimación directa normal o estimación directa simplificada), está obligado a llevar al corriente los llamados libros de registro de ingresos, de facturas emitidas, de gastos, de facturas recibidas y de bienes de inversión y amortizaciones. A menudo, esta actividad es subcontratada y se deja en manos de un experto o gestoría.

En cuanto al cálculo del rendimiento neto de los ingresos percibidos, se pueden descontar una serie de gastos, siempre que se encuentren debidamente justificados y registrados en los libros de registro obligatorios, y siempre que se produzcan durante el ejercicio (año) de la actividad y que sean claramente necesarios para la obtención de ingresos (en este sentido, es motivo habitual de controversias y consultas si un gasto es o no justificable). Algunos de estos gastos son los siguientes:

- Las cotizaciones a la Seguridad Social o las aportaciones a mutualidades del titular de la actividad.

- El importe de los arrendamientos: alquileres derivados del inmueble donde desarrolle su actividad profesional, esto es, donde tenga su consulta.

- Las cantidades satisfechas por servicios de profesionales, como pueden ser los honorarios de administradores, asesores, notarios, abogados, gestores, etc., siempre que estas retribuciones se encuentren relacionadas con su actividad económica.

- Tributos locales, como el Impuesto sobre Bienes Inmuebles (IBI), la tasa de basuras o el Impuesto sobre Actividades Económicas (IAE).

- Gastos de suministros, como pueden ser los de agua, gas, electricidad, internet, etc. relacionados con su actividad. En este caso, en el supuesto 
de desarrollar su actividad en su propia vivienda habitual (desde el año 2018), se permite considerar como gasto deducible el importe resultante de aplicar el $30 \%$ de la proporción de los metros cuadrados de la vivienda que se encuentran destinados a la actividad respecto a su superficie total, salvo que se pruebe un porcentaje superior o inferior.

- Primas de seguros, como los de responsabilidad civil u otros. En el supuesto de primas de seguro de enfermedad del propio profesional, tiene un límite máximo de deducción de 500 euros anuales.

- Gastos de conservación y reparación de los elementos o bienes que utiliza en su actividad.

- Gastos financieros. En este apartado, se pueden incluir los intereses pagados durante el año de los préstamos y créditos solicitados para el desarrollo de la profesión; por ejemplo, la solicitud de un préstamo para la adquisición de la consulta médica.

- Gastos derivados de la asistencia a congresos, cursos, conferencias, seminarios, siempre que se encuentren relacionados con su actividad. De igual modo, son deducibles la suscripción a revistas, libros u otros fondos documentales que sean necesarios para la profesión.

- Cuotas de asociaciones empresariales, cámaras y corporaciones.

- También, desde 2018, tienen la consideración de gasto deducible los gastos de manutención en los que incurre el profesional sanitario en el desarrollo de la actividad económica, siempre que se produzcan en establecimientos de restauración y hostelería y se abonen utilizando cualquier medio electrónico de pago. El importe máximo a deducir, actualmente, se encuentra establecido en 26,67 euros diarios si el gasto se produce en España o 48,08 euros si el gasto se produce en el extranjero, cantidades que, a su vez, se duplican si, como consecuencia del desplazamiento, también se pernocta.

Como puede adivinarse, la variedad de gastos susceptibles de ser justificables es muy amplia y no siempre queda clara ni se puede indicar de forma exhaustiva en la regulación, por lo que, a menudo, tendrá que ser el profesional el que, en caso de ser requerido por las autoridades de la Agencia Tributaria, deba justificar y argumentar que dicho gasto es necesario para el ejercicio de su actividad profesional.

Una vez especificados todos los gastos que se pueden considerar deducibles, se procede a restarlos de los ingresos percibidos en el ejercicio, obteniendo, así, el rendimiento neto de actividades profesionales, cuyo importe hay que incluir en la base imponible general del IRPF (al igual que se indicó más arriba cuando se trataba de rendimientos del trabajo), tributando también a la escala progresiva, que, actualmente, abarca desde un mínimo del $19 \%$ hasta un máximo del $47 \%$, en función de los rendimientos obtenidos durante el ejercicio.

Otro elemento que tener en cuenta es la obligación fiscal en el supuesto de que existan compras a lo largo del año que superen - a un mismo proveedor- el importe de 3000 euros: es la presentación del modelo 347 (se trata de un modelo informativo).

Otra obligación fiscal es la relativa a la presentación de los pagos fraccionados, que se realizan trimestralmente (el conocido modelo 130, que se presenta en los meses de abril, julio, octubre y enero), en el que se produce lo que se denomina «un pago a cuenta» de la futura declaración de la renta.

Asimismo, en el supuesto de tener personal contratado, se tiene la obligación de realizar las correspondientes retenciones por el IRPF en su nómina, e ingresarlas trimestralmente en la Agencia Tributaria (modelo 110, en abril, julio, octubre y enero).

\section{CASUÍSTICA PARTICULAR DE ALGUNOS PROFESIONALES DE LA SALUD A EFECTOS FISCALES}

Otra cuestión que se plantea con frecuencia es el supuesto de ser trabajador por cuenta ajena, pero 
realizar la actividad en clínicas o consultas que no son propias; esto es, cuando el profesional sanitario presta sus servicios, por ejemplo, en una clínica, pero sin ser lo que coloquialmente se llama «falso autónomo». En este caso, los gastos se reducen, puesto que no se asumen todos los gastos derivados del mantenimiento de las consultas, y la obligación que surge es la relativa a la facturación, pues el $100 \%$ de esta irá dirigida a esta clínica. Como consecuencia de ello, en estos casos en los que más del $70 \%$ de los ingresos profesionales se encuentran sujetos a retención, el profesional sanitario no tendrá la obligación de presentar trimestralmente los pagos fraccionados o pagos a cuenta (modelo 130) relativos a su actividad. Igualmente, al no tener personal contratado ni consulta propia, tampoco tiene alquileres, por lo que no tendrá que presentar los impuestos trimestrales que se han indicado en los párrafos anteriores.

Otra posibilidad es que el profesional sanitario preste sus servicios como médico en una sociedad médica de la que es partícipe. En este caso, tiene que declarar los ingresos de su actividad como profesional y no como trabajador asalariado, tal y como establece el artículo 27 de la Ley del IRPF conforme a la consulta de la Dirección General de Tributos de 13 de abril de 2015. En particular, el artículo 27.1 de la Ley del IRPF establece lo siguiente: «No obstante, tratándose de rendimientos obtenidos por el contribuyente procedentes de una entidad en cuyo capital participe derivados de la realización de actividades incluidas en la Sección Segunda de las Tarifas del Impuesto sobre Actividades Económicas, aprobadas por el Real Decreto
Legislativo 1175/1990, de 28 de septiembre, tendrán esta consideración cuando el contribuyente esté incluido, a tal efecto, en el régimen especial de la Seguridad Social de los trabajadores por cuenta propia o autónomos, o en una mutualidad de previsión social que actúe como alternativa al citado régimen especial conforme a lo previsto en la disposición adicional decimoquinta de la Ley 30/1995, de 8 de noviembre, de ordenación y supervisión de los seguros privados».

Como puede verse, la cuestión tributaria de los profesionales del sector de la salud gira en torno a su consideración como asalariados (trabajadores por cuenta ajena) o como autónomos. En este segundo caso, hay que prestar especial atención a la llevanza de los libros contables y de registro, así como a la pertinencia de la deducibilidad de los gastos.

Adicionalmente, hay algunas casuísticas particulares que pueden afectar a determinados profesionales del sector de la salud y que deben ser bien conocidas para poder tener «una convivencia fiscal sana y saludable».

\section{BIBLIOGRAFÍA}

- Agencia Tributaria [Portal]. Disponible en: www.agenciatributaria.es

- Ley 35/2006, de 28 de noviembre, del Impuesto sobre la Renta de las Personas Físicas y de modificación parcial de las leyes de los Impuestos sobre Sociedades, sobre la Renta de no Residentes y sobre el Patrimonio. BOE. 2006;(285).

- Real Decreto 439/2007, de 30 de marzo, por el que se aprueba el Reglamento del Impuesto sobre la Renta de las Personas Físicas y se modifica el Reglamento de Planes y Fondos de Pensiones, aprobado por Real Decreto 304/2004, de 20 de febrero. BOE. 2007;(78). 